\title{
CONFERENCE REPORT TOP2BTM SYMPOSIUM ON HEALTH CARE FOR MEN WHO HAVE SEX WITH MEN (MSM)
}

\author{
K Rebe, $M B C h B, F C P(S A), D T M \& H, D i p H I V$ Man $(S A)$ \\ G de Swardt, $B A(M W)$ \\ H Struthers, $M S c, M B A$ \\ J A McIntyre, $M B C h B, F R C O G$ \\ Anova Health Institute, Johannesburg and Cape Town, South Africa
}

Men who have sex with men (MSM) are at high risk of HIV acquisition and transmission, and country-specific HIV prevalence rates are always higher in MSM than among heterosexual men. South African data confirm this, with reported HIV prevalences of 10.4 - $33.9 \%$ across various studies. Donors and government health planners have recognised the need for targeted programmes that address the high burden of HIV transmission and disease in stigmatised populations such as MSM, as well as other 'most at risk populations' (MARPS) such as commercial sex workers, drug users and displaced refugees. Specific programmes targeting MSM and other MARPS have been included in the South African government's current National Strategic Plan for health care and will feature in the new plan under development.

Until recently, African MSM have been under-researched and underresourced, and this has contributed to their stigmatisation. Fortunately this deficiency has been recognised locally and a number of innovative programmes have been developed to address this. The Top $2 \mathrm{btm}$ symposium on prevention, treatment and care of MSM sought to bring together these programmes to share experiences. The symposium was held in Cape Town, organised by the Anova Health Institute with support from USAID and PEPFAR. The conference attracted 188 delegates from across Africa as well as Europe and North America, including local MSM community representatives, a variety of NGOS, government leaders and health care workers as well as prominent MSM researchers.

Dr Yogan Pillay, South Africa's Deputy Director General of Strategic Planning in the Department of Health, opened the conference on behalf of the Minister of Health. He affirmed government's commitment to implementing targeted HIV and sexually transmitted infection (STI) prevention and treatment programmes for MARPs, including MSM. He highlighted the importance of MSM-targeted HIV testing programmes, considering pre-exposure prophylaxis (PrEP), promoting post-exposure prophylaxis (PEP), encouraging MSM-related research and embracing the concept of antiretroviral treatment as prevention. Consideration is being given to providing state-funded ART to everyone at a CD4 count of 350 cells/ $\mu$ or less; this would be especially beneficial to MSM, since the risk of HIV transmission is much higher during unprotected penileanal sex than during unprotected penile-vaginal sex, and lowering the

\section{ABOUT ANOVA HEALTH INSTITUTE \& HEALTH4MEN}

Health4Men is a special interest programme of the Anova Health Institute, which receives support from USAID / PEPFAR. This programme targets men who have sex with men (MSM) for HIV and STI prevention and treatment. The programme has a strong community focus aimed at improving social support and decreasing stigma experienced by township-based MSM. Health4Men seeks to improve access to health care services and provides education and training for MSM as well as for health care providers who deliver care to MSM clients. Educational programming includes regular training activities and academic symposia. The most recent of these was the Top2btm symposium held in Cape Town in May 2011. viral load of positive MSM is likely to provide a large reduction in HIV transmission.

The conference incorporated four main themes:

1. Understanding the epidemiology of African (and South African) MSM

2. Prevention interventions to address HIV among MSM in Africa

3. Health care services for HIV-positive MSM

4. Research to improve understanding of African MSM.

\section{THE EPIDEMIOLOGY OF MSM IN AFRICA}

The conference proceedings were underpinned by several key concepts in thinking about MSM and HIV/AIDS. First, it is important to recognise that 'MSM' is a medicalised term describing the behaviour of sex between men; it does not describe a particular identity or population group. MSM are extremely diverse and probably have many differences from each other and only one main commonality, having sex with other men. MSM vary in the way they conduct their lives and in the sex that they have (for example, not all MSM engage in anal sex). They may identify as heterosexual, homosexual or bisexual. They may possess a masculine or feminine identity and they may identify with 'gay' or mainstream culture.

Related to this, it is common throughout Africa to find MSM who identify as heterosexual and are even married, appear masculine but sometimes have sex with men in addition to women. These MSM are particularly difficult to reach with traditional health programmes, as they are in many ways invisible in mainstream society.

Second, MSM experience a range of barriers to accessing health care, not least stigma and prejudice from health care providers themselves. MSM often receive counselling that is inappropriate to the lives they lead. For example, HIV prevention messages aimed solely at heterosexuals may ignore the risks of HIV transmission associated with anal sex. MSM are concerned about the double stigma they may experience in health centres relating to their sexual orientation and their HIV status, and are often reluctant to seek care.

With this background, the keynote address entitled 'Time to act: Responding to the HIV pandemic among MSM' was delivered by Professor Chris Beyrer of Johns Hopkins Bloomberg School of Public 
Health (USA). Systematic reviews of HIV in low- and middle-income countries from which data are available consistently show that MSM are at higher risk of HIV compared with the local heterosexual population. Aggregate HIV prevalences among African MSM are reported to range from $8.8 \%$ in Sudan to $32.9 \%$ in Zambia. Many of these studies were performed using respondent-driven or 'snowball' sampling and therefore may not be generalisable; however, they do highlight the concentrated MSM HIV epidemic in countries where there is also a heterosexual epidemic. In addition, pooled data from studies in Malawi, Botswana and Namibia demonstrate health provider stigma: $5.1 \%$ of MSM sampled report being denied health care services because of their sexual orientation, and $22.3 \%$ reported 'any discriminatory event' based on sexuality. Provider stigma is just one of the challenges currently facing MSM.

For HIV transmission to occur, transfer of an HIV-containing fluid needs to gain entry into a new individual. The anal mucosal lining is thin, does not self-lubricate and is more liable to mucosal tears than vaginal mucosa. Biologically, unprotected anal sex, particularly receptive anal sex, carries a high risk of transmitting HIV (estimated to be approximately $1.4 \%$ with each episode, which is roughly 18 times higher than for vaginal sex). Condoms and other HIV-risk reducing interventions are therefore extremely important for MSM.

Individual-level HIV risks include unprotected anal sex, high numbers of sex partners, and injecting and non-injecting drug use. Structural level risks for MSM relate to stigma, discrimination and human rights concerns. A study in Namibia, Malawi and Botswana showed selfreporting of human rights abuse to be high; for example, 5.1\% of MSM studied had been denied health services based on their sexuality and $23 \%$ reported any form of discrimination. This and similar studies show the difficulty faced by MSM trying to access healthcare in stigmatised and even criminalised environments.

\section{HIV PREVENTION INTERVENTIONS FOR MSM}

A number of presentations addressed HIV prevention for MSM. In subSaharan Africa the incidence of HIV is declining among heterosexual people but continues to rise in MSM, illustrating the need for innovative prevention programmes. Professor Linda-Gail Bekker from UCT called for a time of 'highly active HIV prevention'. In particular, the role of ART as prevention is gaining ground, and this was visible during discussion at the conference. Evidence cited included the Pre-Exposure Prophylaxis Initiative (iPrEx) and the recently released results of the HPTN 052 trial.

iPrEx recruited 2499 HIV negative high-risk MSM and randomised them to receive either Truvada or placebo daily in addition to risk reduction counselling, monthly HIV testing, condom and lube provision and treatment of STIs. Most recruitment occurred in South America, but 90 MSM (4\%) were recruited at a Cape Town site. Results showed a $44 \%$ reduction in HIV infections in the treatment arm and there was a significant dose-response relationship with better adherence associated with increased protection. Guideline documents for the use of PrEP are available and should be included as an option in the 'prevention package' for MSM.

The HPTN 052 trial recruited 1763 discordant couples (only 3\% MSM) and randomised them to either early (CD4 count 350 - 550 cells/ $/$ l) or late (CD4 <250) ART initiation for the positive partner. The trial found that earlier treatment decreased HIV transmission by $96 \%$ over the duration of follow-up. The study was stopped early by its monitoring board, and we await full publication. Early indications are good that ART did provide significant protection in heterosexual discordant couples, but the study was underpowered for MSM.
Other prevention strategies discussed by speakers for inclusion on the prevention 'menu' for MSM include:

Biomedical. In addition to PrEP, biomedical prevention options for MSM include post-exposure prophylaxis (PEP), innovative marketing and distribution of condoms (including the female condom for anal sex) and sexual lubricants, STI screening and treatment. Rectal microbicides are desirable but are not yet fully developed or proven to be effective. Medical male circumcision has not been shown to confer protection for MSM, except perhaps if they are exclusively the penetrative partner in anal sex or have concurrent sexual relationships with women, where the infective risk is from penile-vaginal sex. Programmes targeting MSM who use substances, particularly alcohol and crystal methamphetamine, are required, as are needle exchange programmes.

Behavioural. Counselling programmes to modify high-risk behaviour were emphasised. 'Serosorting' and 'seropositioning' (choosing sexual partners on the basis of their HIV status, or deciding on insertive or receptive anal intercourse depending on partner status) were discussed and may be of value but could be construed as 'sero-guessing' in areas where MSM do not know their status or misinform potential sex partners.

Structural. Advocacy is needed to decrease stigma and discrimination from general society and from health care providers. Dr Patrick Sullivan emphasised the role of using technology, specifically Internet-based and mobile phone-based platforms in prevention. Related to this, Health4Men announced a new mobi site where MSM in South Africa can access HIV information and ask questions from their cellphones (http://h4m.mobi).

\section{HEALTH CARE SERVICES FOR HIV-POSITIVE MSM}

Professor James McIntyre detailed the history of the Anova Health Institute's Health4Men project that led to the establishment of holistic sexual health and HIV prevention and treatment services for MSM in South Africa. Two MSM-targeted clinics operate in Cape Town (the Ivan Toms Centre for Men's Health) and Soweto (the Simon Nkoli Centre for Men's Health), with a satellite clinic in Pretoria. These clinics are supported by USAID/PEPFAR and the Department of Health and are at the forefront of health provision for MSM in Africa.

The Ivan Toms Centre for Men's Health in Cape Town has been operating for more than 2 years. The clinic offers a primary-care level, holistic package of HIV and STI prevention and treatment services, including the provision of government-funded ART for HIV-positive MSM who qualify for treatment according to national guidelines. The clinic includes an extensive mental health component in collaboration with the Department of Psychiatry at Groote Schuur Hospital. Approximately 3000 clients have utilised the clinic's services so far. About half of these clients are HIV-positive and approximately 500 are currently receiving ARVs. The clinic addresses a large non-HIV STI burden, with syphilis and human papillomavirus infection being particularly common. For example, approximately $15 \%$ of individuals screened at the clinic are positive for syphilis infection.

This clinic is unique in not marketing itself as a 'gay clinic' or an 'HIV clinic.' The client cohort includes both negative and positive people, some of whom receive ART, and some remain in wellness programmes prior to initiating treatment. This model has advantages in terms of enabling health-seeking behaviour of MSM, as individuals are not identifiable as HIV-positive because of the clinic they attend. Many attend for other STIS, or for counselling or other services. 
MSM treatment challenges were addressed in a number of sessions at the conference. The importance of training of health care workers to decrease homoprejudice in the health sector was stressed. Homoprejudice from the health sector acts as a structural barrier to health care access for MSM. Already organisations in South Africa, including the Anova Health Institute and the Desmond Tutu HIV Foundation, are conducting training programmes for health care providers to address this.

The conference discussions emphasised that there are some considerations that must be borne in mind when providing ART to MSM. Some groups of MSM are very body conscious, and adherence to drugs causing lipo-atrophy may be low. Similarly, MSM who develop erectile dysfunction may default protease inhibitors if these are perceived as contributing to the problem. Mental health and drug use, and how these may influence adherence, also need to be considered when initiating ART in HIV-positive MSM.

\section{MENTAL HEALTH}

The need to incorporate mental health services into the package of care for MSM was highlighted by Dr Kevin Stoloff from the Department of Psychiatry at Groote Schuur Hospital. His presentation, based on literature review and clinical experience at the Ivan Toms Centre for Men's Health, stressed that high levels of anxiety, depression, personality disorders, internalised homoprejudice, substance abuse and other mental health challenges make adherence support vital for MSM. These same mental health challenges may precipitate or enhance risk taking among MSM.

\section{DRUG USE}

Drug use is common, and some communities in South Africa are experiencing an explosive increase in crystal methamphetamine use. It is recongised that a large percentage of incident HIV infections in the developed world are related to crystal methamphetamine use, and the same may be true of some groups of South African MSM. Crystal methamphetamine use lowers inhibitions, increases risky sexual behaviours and may increase biological susceptibility to HIV infection. The drug can also be injected, which raises concerns about needle sharing and transmission of other blood-borne viruses such as hepatitis B and C. Crystal methamphetamine and other recreational drugs can have unanticipated drug-drug interactions and sideeffects for HIV-positive individuals taking antiretroviral medications, making treatment of such people difficult from medication choice and adherence perspectives.

\section{ANAL INTRA-EPITHELIAL NEOPLASIA}

An under-recognised health care problem for MSM is anal intraepithelial neoplasia (AIN) and anal cancer. A presentation on this issue highlighted the complete absence of screening and treatment services in South Africa. AIN is a precursor to anal cancer and parallels cervical intra-epithelial neoplasia in women. AIN is HPV-associated and may lead to cancers that involve peri-anal and anal skin. Screening for and early detection and management of AIN may prevent anal cancers. Many experts now advocate for the inclusion of AIN screening in routine care of MSM. Not doing so represents a missed opportunity to prevent serious malignancies.

\section{TRANSGENDER ISSUES}

Dr Anita Radix from the Callen-Lorde clinic in New York provided valuable insights into the health care needs of transgender people (TG). TG face individual and structural barriers to health care access that are sometimes different from those experienced by MSM. There is a dearth of services for TG in Africa and many health care providers lack the skills to manage complex psychological and medical issues, including management of complex drug-drug interactions between hormones and ART. Health care worker sensitisation and education programmes are required.

\section{RESEARCH}

Professor Carolyn Williamson, a medical virologist at the University of Cape Town, discussed HIV subtypes that circulate in Cape Town. The predominant HIV subtype in MSM in developed nations is subtype $B$, contrasting with heterosexual epidemics where subtype C predominates, as in South Africa. Phylogenetic studies performed with 147 HIV samples from mixed urban and rural South African MSM showed approximately $80 \%$ to be subtype C, $13 \%$ to be subtype B and the balance to consist of various other subtypes. This may have consequences for future vaccine research for MSM in Africa, as a vaccine directed primarily against subtype B virus may be less effective in a subtype Cor recombinant subtype epidemic.

\section{CLINICAL SKILLS FOR HEALTH SERVICE PROVIDERS}

Two workshops were included in the symposium, both aimed at improving the clinical skills of health providers who service MSM. The first workshop addressed discussing sex and taking a sexual history in a clinical setting. Participants received guidance in this arena and learned skills were reinforced by role-play activities with concrete examples of sexual histories taken from MSM who had recently attended the Ivan Toms Clinic. The second workshop addressed the use of post-exposure prophylaxis. Workshop attendees received background information and practical advice about the administration of PEP and for including this HIV transmission reducing intervention into the scope of services provided at their clinics. Key learning points from each of these workshops are presented in Boxes 1 and 2.

\section{SUMMARY}

In summary, the Top2Btm conference offered a full and broad-ranging programmme with topics covering issues in epidemiology, prevention, treatment and research relating to MSM in Africa.

Throughout the conference the diversity of MSM communities in South Africa was stressed, as was the need for innovative and tailored programmes to address the health needs of these often hidden communities. Clinics such as the Ivan Toms Centre for Men's Health in Cape Town have developed expertise and a model for disseminating the knowledge and skills required to achieve the aim of targeted health care for MARPS, including MSM, in the country's National Strategic Plan for Healthcare.

\section{Box 1. Taking a sexual history in a clinical setting}

- Do not assume heterosexuality among men attending HIV clinics.

- Ensure privacy and confidentiality of information.

Ensure staff training to enable them to confidently address a broad range of sexualities and sexual problems.

- Explain the context of the sexual history in terms of identifying health risks and individualising an HIV risk reduction plan.

- Build rapport with patients by asking generalised questions.

- Thereafter, ask all clients if they have sex with women, men or both.

- Use local colloquial language that is accessible to clients.

Do not moralise about clients'sexual activities. The aim is to normalise all consensual sex while identifying areas where HIV transmission risk can be reduced. 
